\title{
Prosthodontic management of mandibular defects subsequent to head and neck cancer- A review article
}

\author{
Avanti Merchant ${ }^{1 *}$, Rubina Tabbassum ${ }^{2}$, Gaurang Mistry ${ }^{3}$, Charushila Sardar $^{4}$, Gauri Merchant ${ }^{5}$ \\ ${ }^{13 r d}$ Year Postgraduate Student, ${ }^{2,4}$ Professor, ${ }^{3,5} \mathrm{HOD},{ }^{1-4}$ Dept. of Prosthodontics, ${ }^{5}$ Dept. of Oral Health and Advanced Dentistry, ${ }^{1-4}$ DY Patil \\ University School of Dentistry, Navi Mumbai, Maharashtra, ${ }^{5}$ Sir H. N. Reliance Foundation Hospital, Mumbai, Maharashtra, India \\ *Corresponding Author: Avanti Merchant \\ Email: avantimerchant19@gmail.com
}

\begin{abstract}
Cancer of the mandible is seen to be one of the most common forms of cancer of the head and neck region. Due to lifestyle changes, consumption of alcohol and tobacco a large spike in the number of reported cases has been seen. Squamous cell carcinoma of the tongue and mandible is one of the most debilitating forms of cancer and is seen to take not only a physical but also a mental toll on the patients psychological status. This article hereby tries to highlight the importance of maxillofacial rehabilitation of defects of the mandible subsequent to surgical intervention.
\end{abstract}

Keywords: Maxillofacial rehabilitation, Hemimandibulectomy, Palatal Augmentation Prosthesis, Guide Plane Prosthesis.

\section{Introduction}

Squamous cell carcinoma of the tongue and mandible is one of the most aggressive forms of cancers to be reported in literature. In most cases these lesions are ignored and detected at a very delayed stage, where prognosis of the disease is usually poor. Treatment of such patients usually requires complete excision of the tumour, along with removable of vital structures such as muscles and lymph nodes of the area. Hemimandibulectomy surgery is one which is very disfiguring and disabling. Due to loss of large amounts of bone, muscles and teeth these surgeries not only incapacitate the patient physically but also play on the mentality of the patient often leading to conditions such depression. Maxillofacial rehabilitation greatly helps to improve the quality of life of these patients. By following an interdisciplinary approach with various medical teams we can try to help regain bodily functions such as speech, mastication, swallowing, and enhance the esthetics of the patient. Maxillofacial rehabilitation has not just a role in improving the physical health of the patient, but helps to regain the self-image and confidence of the patient to help him live as normal a life as possible.

\section{Cancer of the Mandible}

Cancer of the mandible is one of the most rampant and widely existing cancers of the head and neck region mainly due the consumption of tobacco in smoke and smokeless forms. It is seen to be one of the leading causes of death and one of the most pervasive forms of cancer in the Indian subcontinent. It is important to note that during the treatment of such patients emphasis must be placed not only on the early surgical removal of the tumour but the rehabilitation of the patient as well to improve their overall quality of life.

Hemimandibulectomy surgery can have several consequences in the future. The most common complication is deviation of the unaffected jaw to the surgical side. Due to the loss of the attachments of the muscles of mastication on the resected side, there is a frontal plane rotation of the mandible that is observed during forceful closure. ${ }^{1}$ When viewed from the frontal plane, teeth on the surgical side of the mandible move away from their opposing maxillary teeth after their initial contact on the non-surgical side is achieved. During surgery structures such as the tongue and teeth might not be spared and hence glossectomy defects have to be accounted for as well. Functions such as speech and mastication might be greatly hampered during this process; hence every effort must be made by the prosthodontist to help improve the quality of life of these patients.

\section{Classification of Mandibular Defects}

Numerous classifications for defects of the mandible exist, the most widely followed one are Cantor and Curtis Classification, followed by Jewers Classification.

Table 1: Cantor and Curtis classification of mandibular defects

\begin{tabular}{|l|l|l|}
\hline Class & Type of defect & \\
\hline I & Radical alveolectomy with preservation of \\
II & mandibular continuity. \\
III & Lateral resections of the mandible distal to the canine \\
IV & area. \\
V & $\begin{array}{l}\text { Resections of the mandible to the midline. } \\
\text { VI }\end{array}$ & $\begin{array}{l}\text { Lateral resections which were surgically } \\
\text { reconstructed with bone. }\end{array}$ \\
& $\begin{array}{l}\text { Anterior mandibular defects with bone graft } \\
\text { reconstruction. } \\
\text { Anterior mandibular defects without surgical } \\
\text { reconstruction. }\end{array}$ \\
\hline
\end{tabular}

Table 2: Jewers classification of mandibular defects

\begin{tabular}{|l|l|}
\hline Class & Type of defect \\
\hline $\mathrm{C}$ & Central defects of mandible from canine to canine. \\
$\mathrm{L}$ & $\begin{array}{l}\text { Lateral defects extending from midline to the } \\
\text { condyle. }\end{array}$ \\
$\mathrm{H}$ & $\begin{array}{l}\text { Hemi-mandibular defects including ' } L \text { 'defects of the } \\
\text { condyle. }\end{array}$ \\
\hline
\end{tabular}




\section{Prosthodontic Rehabilitation with Different Treatment Modalities Palatal Augmentation for Glossectomy Defects}

The tongue, particularly its posterior lateral aspect, is a common site for oral carcinomas. Frequently, the treatment involves surgical excision, radiation therapy, or both. In patients with extensive lesions, the resections may include the floor of the mouth and the mandible in addition to the tongue. With limited resections, where $45 \%$ or less of the tongue is removed, patients have little functional impairment. However, in patients with more extensive resection, impairment of mastication, deglutition, and speech may occur.

A Palatal augmentation prosthesis is beneficial for the rehabilitation after glossectomy of patients with head and neck cancer. Its use enables patients to perform compensatory movements of the tongue in relation to the surrounding structures, movements that are essential for articulation and the oral preparatory and oral phases of deglutition. The prosthesis is fabricated by adding bulk of acrylic on the palatal aspect of the patient which is moulded according to functional movements of the patient. This prosthesis in conjunction with a speech therapist and pathologist will aid to improve the speech of the patient to the utmost level.

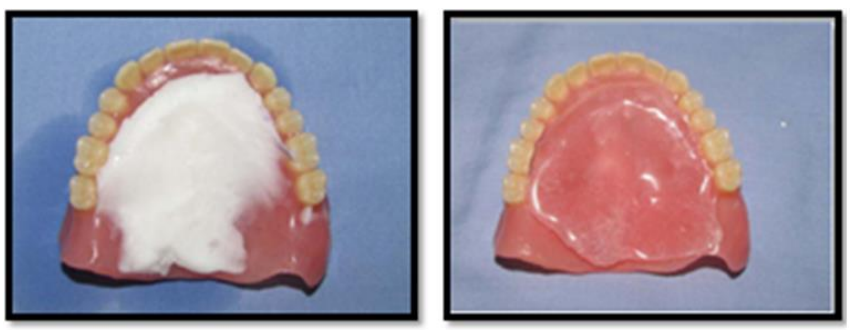

Fig. 1: Palatal augmentation prosthesis

\section{Guide Plane Prosthesis}

The unilateral loss of mandibular continuity due to surgery or trauma results in mandibular deviation toward the defect side with lack of occlusion. Unlike the dentulous patients, edentulous patients are difficult to retrain for mandibular movement and many times may never achieve proper maxillomandibular relationships required for optimum mastication and appearance. ${ }^{2}$ The use of a guide plane prosthesis is one such treatment modality that can be used for the correction of this deviation. A maxillary acrylic inclined guiding plane prosthesis could be fabricated to assist muscle retraining as acrylic indentations created by the bite of the patient help to move and hold the mandible in maximum intercusspation. When the maxillomandibular relationship improves a more definitive treatment plan can follow. The retraining of the residual mandibular muscles would permit occlusion of remaining natural teeth or control of residual edentulous segments to provide for the reasonable placement and acceptable occlusion of the artificial teeth. To attain acceptable occlusal contacts with the remaining natural teeth on unresected side, a flat, wide twin maxillary occlusal table on resected side can be made to compensate for the deviation that can provide a surface against which the artificial teeth of the residual segment can occlude.

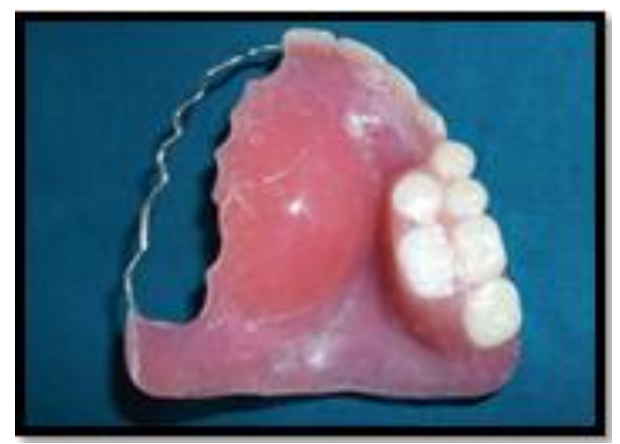

Fig. 2: A guide plane prosthesis

\section{Implants in Mandibular Rehabilitation}

A proper choice of reconstruction techniques in combination with implant supported or retained prosthodontics probably can attribute to better functional results in the oral rehabilitation of patients with head and neck cancer. As a first effect, implants are used with increasing frequency for prosthetic support in patients who are treated for malignancies in the lower region of the oral cavity. ${ }^{3}$ This includes reconstruction of the mandible and insertion of implants in patients who have been treated with radiotherapy as well. In patients with large defects, bone augmentation procedures might also be required. ${ }^{4}$ Free fibular grafts are the grafts of choice for the same. One of the complications that might occur is that irradiated sites might be at significant risk for tissue necrosis and loss of implants, if subjected to implant surgery. Thus, the appropriateness of using implants in irradiated patients has been seriously questioned. Because of the radiation hazards mentioned, it might be reasonable to place implants prior to postoperative radiotherapy, preferably simultaneously with ablative surgery. From literature, it can be derived that implant-retained mandibular overdentures can strongly reduce the problems with stability and retention of a denture and relief to the underlying soft tissues, particularly if the occlusal load is borne by the implants. In some cases, the soft tissues might be surgically reconstructed and continuity can be re-established with a reconstruction plate or a bone graft with an insufficient volume (e.g. costal grafts) for reliable implant placement. In irradiated patients a minimum of four implants is recommended. These implants should be placed in optimally spaced locations for the best possible spread of occlusal loading. Implant therapy is now the gold standard treatment for the rehabilitation of patients undergoing hemimandibulectomy. Implants are seen to greatly improve the chewing efficacy of patients, and in cases of removable appliances help to ameliorate the stability as well as the retention of the prosthesis by considerable amounts. 


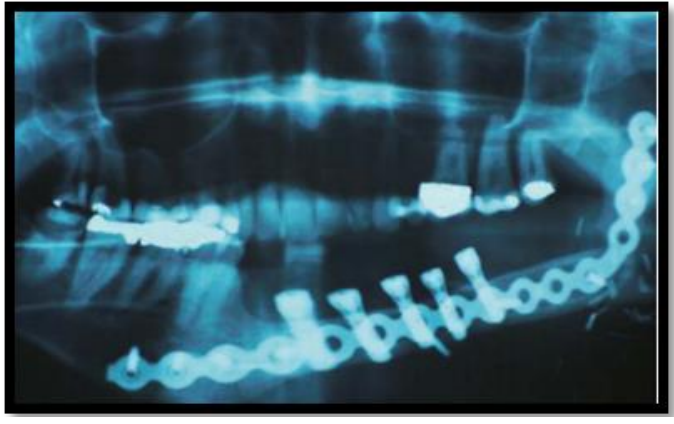

Fig. 3: Implant therapy in hemimandibulectomy patients

\section{Recent Advances in Mandibular Rehabilatation}

With the advent of CAD CAM technology in the field of dentistry, many positive changes and new approaches have been advocated in the field of maxillofacial prosthodontics as well. CAD CAM dentistry enables us to conduct surgical planning and 3D printing of oral structures prior to actually conducting surgery. An entire hemimandibulectomy surgery can be planned and viewed on a software, followed by printing of the replacement of the defect site. This technology helps us to rehabilitate the patient on the very same day, and spares the surgeon from conducting any subsequent surgeries in the future. On the day of the surgery the patient will receive a replace jaw, along with teeth which will be printed in a laboratory prior to surgical intervention.

\section{Conclusion}

Head and neck cancer and its treatment can have a profound effect on the patient's physical, functional and emotional well-being, especially decreasing the Quality of life. ${ }^{5}$ Quality of life evaluations have increasingly become an important supplement and diagnostic aid in the interpretation of information in head and neck cancer treatment. ${ }^{6}$ It is important to treat these patients in a holistic manner and not only provide maxillofacial rehabilitation but also provide psychological intervention to help boost the morale of the patient and instil in them a sense of confidence to overcome the ravages of post cancer therapy.
Overall, maxillofacial rehabilitation leads to significant improvement in all impaired functions and has a positive change on the Quality of life. Prosthetic rehabilitation can play a key role in not only improving the physical attributes of the patient but also instil in them the confidence to improve their self-image and aid in re-socialization.

\section{Source of Funding}

None.

\section{Conflict of Interest}

None.

\section{References}

1. Murphy BA. Advances in quality of life and symptom management for head and neck cancer patients. J Oncol. 2009;21(2):42-7.

2. Aramany MA, Downs JA, Beery QL. Prosthodontic rehabilitation for glossectomy patients. J Prosthet Dent. 1982;48:78-8

3. Curtis TA, Griffith MR, Firtell DN. Complete denture prosthodontics for the radiation patient. J Prosthet Dent. 1976;36:66-76.

4. Cantor R, Curtis TA, Shipp T, Beumer J, Vogel BS. Maxillary speech prostheses for mandibular surgical defects. J Prosthet Dent. 1969;22(1):249-53.

5. Bidra AS, May GW, Tharp GE, Chambers MS. Pterygoid implants for maxillofacial rehabilitation of a patients with a bilateral maxillectomy defect. J Oral Implantol. 2011;37(2);81-7.

6. Bilbulian Ah Springfield, Charles C. Facial prosthetics, Current state of craniofacial prosthetic rehabilitation, Int J Prosth. 1973;26(1):57-67.

How to cite this article: Merchant $A$, Tabbassum $R$, Mistry G, Sardar C, Merchant G. Prosthodontic management of mandibular defects subsequent to head and neck cancer- A review article. Int J Oral Health Dent 2020;6(1):5-7. 